\title{
Les protéines Ras et GAP, des relais sur la voie de transmission du signal passant par l'activation de tyrosine kinases
}

De nombreux facteurs de croissance et quelques hormones se lient à des récepteurs dont la partie carboxyterminale intracellulaire possède une activité de tyrosine kinase : c'est le cas des récepteurs de l'insuline, d'IGF1 (insulin growth factor 1), d'EGF (epidernal growth factor) de PDGF (platelet-derived growth factor), de CSF1 (colony stimulating factor 1), etc. De plus, existent également des tyrosine kinases sans domaine extracellulaire, dont des archétypes sont $\mathrm{p} 60^{\text {scr }}$ et $\mathrm{p} 56^{\text {lck }}$. Les uns et les autres sont impliqués dans des phénomènes de stimulation de la division cellulaire ou de différenciation, suivant les contextes. Par ailleurs, nombreux sont les gènes codant pour ces molécules qui sont les équivalents cellulaires d'oncogènes rétroviraux. Les oncogènes ras sont des petites protéines $G$ appartenant à une grande famille multigénique codant pour des protéines aux fonctions multiples [1, 2]. Jusqu'à il y a peu, on ignorait complètement les liens précis unissant les proto-oncogènes ras aux différentes voies connues d'activation de la croissance cellulaire. En revanche, d'importants progrès avaient été faits concernant la régulation de l'activité des protéines Ras : Ras est inactive sous sa forme liée au GDP et active sous sa forme liée au GTP. Ras-GTP interagit avec des protéines GAP (GTPase activating protein) qui stimulent très fortement son activité GTPasique endogène, induisant donc l'inactivation de la protéine Ras. La transition RasGDP $\rightarrow$ Ras-GTP est catalysée par des facteurs d'échange, encore appelés GDS (GDP dissociation stimulating protein) et pourrait être inhibée par des protéines de type GDI (GDP dissociation inhibiting protein), à vrai dire beaucoup moins bien connues, voire incertaines $\mathrm{m} / \mathrm{s} n^{\circ} 5$, vol. 8, mai 92 pour ce qui est des protéines p21 ras. Chez la levure, deux gènes, $C D C 25$ et SDC25 semblent être des facteurs d'échange agissant sur les gènes $R A S$ de ce micro-organisme $\left(\mathrm{m} / \mathrm{s} n^{\circ} 7\right.$, vol. 6 , p. 703).

Très récemment, nous avons rapporté les expériences du groupe de McCormick concluant que la protéine p120-GAP se comportait, sur certains systèmes, non seulement comme un inhibiteur de Ras, mais aussi comme un relais indispensable à son action sur des cibles situées plus en aval $(\mathrm{m} / \mathrm{s}$ $n^{\circ} 4$, vol. 8, p. 388).

Ras et tyrosine kinases (figure 1)

- La cascade bos/sevenless chez la drosophile

Toute une série de résultats a maintenant démontré que la voie de signalisation passant par les tyrosine kinases nécessitait l'intervention du système Ras/GAP. Les travaux les plus démonstratifs sont probablement ceux portant sur le contrôle génétique des cascades d'événements intervenant au cours du développement. Chez la drosophile, le gène sevenless est indispensable à la différenciation d'un type de photorécepteurs de l'œil, les cellules R7. La protéine Sevenless est un récepteur transmembranaire de type tyrosine kinase. Il est stimulé par le produit du gène bos (bride of sevenless), une protéine transmembranaire synthétisée dans les cellules R8 qui sont ainsi indispensables à la différenciation de la population de neurones R7. Début 1992, deux nouveaux gènes codant pour des protéines situées en aval de cette voie de signalisation étaient détectées : tout d'abord le gène Sos (son of sevenless) dont la séquence révéla qu'il codait pour une protéine similaire à CDC 25 de Saccharomyces cerevisiae, c'est- à-dire probablement pour un facteur d'échange GDS. Des mutants avec perte de fonction du gène Sos ont de très importants troubles du développement, dépassant la non-différenciation des neurones R7 [3]. On découvrit ensuite que la différenciation des cellules R7 à la suite de la stimulation de la protéine Sevenless nécessitait, en aval, un gène Ras-1. L'introduction d'un gène Ras-1 activé par une mutation portant sur le codon 12 , ce qui lui fait perdre son activité GTPasique, est capable de compenser des mutations avec perte de fonction des gènes bos et sevenless [4]. Puis, étaient décrits des mutants Gap-1 mimant l'activation constitutive du récepteur Sevenless ou la transformation par un gène Ras-1 activé. Gap-1 code pour une protéine de type GAP [5]. Cette remarquable série de travaux définissait donc une hiérarchie bos $\rightarrow$ sevenless $\rightarrow$ Sos $\rightarrow$ Ras-1 $\rightarrow$ Gap-1 qui peut s'écrire également facteur de croissance et de différenciation $\rightarrow$ récepteur tyrosine kinase $\rightarrow$ facteur d'échange $\rightarrow$ protéine Ras $\rightarrow$ protéine GAP. A noter que dans l'exemple signalé ci-dessus, la protéine Gap-1 se comporte uniquement comme un régulateur négatif de Ras-1 puisque sa perte de fonction équivaut à une activation constitutive de Sevenless ou de Ras-1. Toute cette cascade d'activation pourrait aboutir au produit du gène sina (seven in absentia), une protéine impliquée dans la différenciation des photorécepteurs R7 [6]. Quoique de phénotype différent de sevenless, les mutants Roughened présentent eux aussi des malformations oculaires et sont une illustration supplémentaire du rôle de Ras dans des processus de différenciation. Le gène Roughened, constitutivement activé chez les mutants, code en effet pour un équi- 


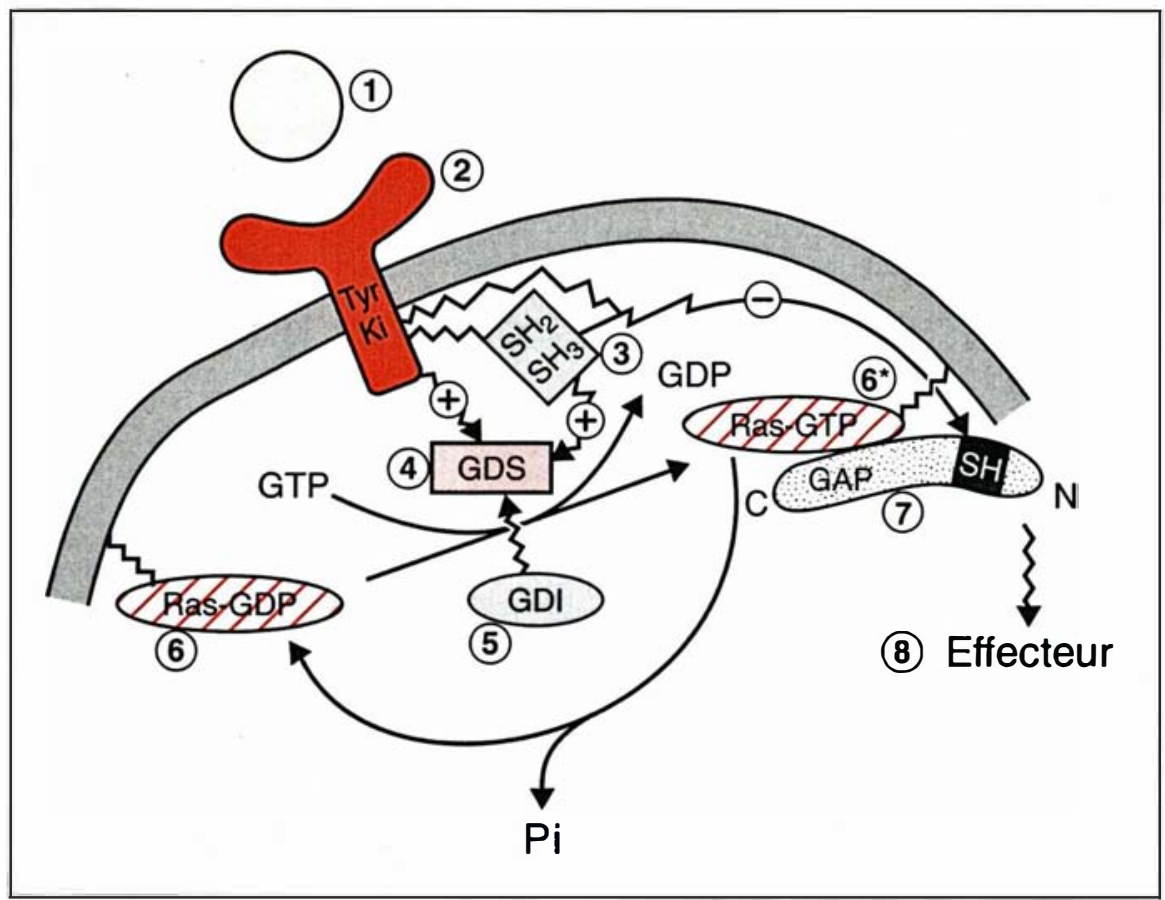

Figure 1. Schéma de différentes étapes de voies de signalisation empruntant des récepteurs à activité tyrosine kinase. Ce schéma représente les facteurs de croissance ou morphogènes (par exemple Bos) reconnaissant un récepteur tyrosine kinase (par exemple, Sevenless, Torso, let-23, récepteur de l'insuline, etc.). Dans certains cas, l'autophosphorylation sur les tyrosines induites par l'occupation du récepteur pourrait faciliter l'interaction avec une protéine intermédiaire à domaines $\mathrm{SH} 2$ et $\mathrm{SH} 3$ (par exemple sem-5) qui serait elle-même reliée à des étapes situées plus en aval. Celles-ci pourraient être le facteur d'échange GDS (par exemple Sos ou CDC 25), qui serait activé, ou bien des protéines GAP qui seraient inactivées. Le facteur inhibant l'échange, GDI, n'est correctement caractérisé ni sur le plan fonctionnel, ni sur le plan moléculaire. L'activation des facteurs d'échanges ou l'inactivation de la GTPase activating protein provoquerait l'accumulation de la forme active de Ras liée au GTP qui interagirait avec certaines protéines GAP. Cette interaction transmettrait plus en aval un signal vers les cibles de cette voie d'induction de la prolifération qui serait rétrocontrôlée grâce à l'activation de la dégradation du GTP en GDP. Certaines protéines GAP pourraient jouer uniquement ce dernier rôle de régulateur négatif et ne pas intervenir dans la transmission du signal. Des facteurs transcriptionnels tels que, probablement, le produit du gène sina ou le complexe AP1 pourraient constituer les cibles finales couplant la mise en jeu d'un programme de croissance ou de différenciation à la modification de l'expression des gènes nécessaire à l'exécution de ce programme. (1) ligand; facteur de croissance; hormone morphogène (Bos). (2) récepteur tyrosine kinase (Sevenless, Torso, Let 23). (3) protéines intermédiaires, Sem 5, p95vav. (4) facteur d'échange; GDS, SOS, CDC25. (5) facteur inhibant l'échange; GDI. (6) petite protéine G Ras inactive et activée*, Let 60. Les protéines $G$ sont fixées à la membrane par un résidu farnésyl. (7) protéine GAP, NF1. (8) cibles effectrices ultimes, probablement facteurs de transcription (Sina, AP1).

valent de Rap-1/Krev-1, un antagoniste supposé de p21 ras [7] $\left(\mathrm{m} / \mathrm{s} n^{\circ} 4\right.$, vol. 5, p. 260).

- La cascade let-23/let-60 chez Caenorhabditis elegans

Chez le nématode Caenorhabditis elegans existe une voie de signalisation simiagissant en cascade : il s'agit du contrôle du développement des structures vulvaires et, notamment, de la migration des myoblastes vulvaires. En 1990, était décrit le gène let-23, codant pour un récepteur de type tyrosine kinase et nécessaire à l'induction de la différenciation vulvaire [8]. Peu après, au cours de la même année, le gène let-60 dont l'activité est indispensable à l'activation de let-23 était cloné. let-60 code pour une protéine de type Ras et comporte des mutations avec perte ou gain de fonction, ces dernières entraînant un phénotype " multivulvaire" [9]. Enfin, très récemment, a été caractérisé le gène sem-5 dont l'intégrité est également indispensable à la différenciation via le récepteur let 23. La séquence de ce gène montre qu'il code pour une protéine uniquement constituée de domaines de type $\mathrm{SH} 2$ et $\mathrm{SH} 3$ [10]. $\mathrm{SH}$ signifie sarc homology et se retrouve dans toute une famille de protéines interagissant avec des phosphotyrosines et des protéines du cytosquelette. $\mathrm{SH} 2$ se lie fortement à des phosphotyrosines faisant partie de séquences peptidiques très spécifiques alors que $\mathrm{SH} 3$ interagirait avec des protéines du cytosquelette. De tels domaines $\mathrm{SH} 2$ et SH3 sont retrouvés au niveau de diverses protéines capables de s'associer à des récepteurs tyrosine du type du récepteur de l'EGF : tel est le cas de la phospholipase $\mathrm{C} \gamma$, d'une sous-unité de la phosphatidyl-inositol 3-kinase et de la protéine Raf, une sérinethréonine kinase [11]. Raf-1 est hyperphosphorylée sur des sérines et des thréonines lors de la stimulation de nombre de récepteurs à activité de tyrosine kinase. Sans que l'on connaisse les mécanismes de cette phosphorylation, des travaux publiés en mars 1992 montrent que, au moins dans les cellules à différenciation neuronale PC12, la fixation du NGF (nerve growth factor) par son récepteur (le produit du proto-oncogène $t r k)\left(\mathrm{m} / \mathrm{s} n^{\circ} 6\right.$, vol. 7, p. 620) entraîne une phosphorylation de $\mathrm{Raf}-1$ relayée par Ras : en effet, un mutant transdominant de Ras (une molécule Ras dont la sérine 17 a été remplacée par une asparagine et qui fixerait le facteur d'échange GDS avec une forte affinité) bloque l'effet de NGF sur la phosphorylation de Raf-1 [12]. Pour en revenir au gène sem 5 , on peut supposer qu'il est un relais entre différentes molécules de la voie de signalisation commandée par let 23. Il pourrait, par exemple, coupler l'autophosphorylation du récepteur tyrosine kinase, produit de let 23 , à un effecteur plus aval, par exemple une molécule de type GDS qui serait activée ou une GTPase activating protein qui 
serait inhibée (figure 1). Ce rôle présomptif de sem 5 chez C. elegans rappelle celui proposé il y a peu pour l'oncogène vav chez les mammifères : son produit, la protéine p95 vav, contient en effet des domaines $\mathrm{SH} 2$ et SH3 et, après stimulation, s'associe à des récepteurs de facteur de croissance, au TcR ( $T$ cell receptor) et au récepteur des IgE sur les basophiles. La phosphorylation de $\mathrm{p} 95^{\mathrm{vav}}$ sur des résidus tyrosine ainsi induite lui permettrait de transmettre le signal à d'autres cibles $[13,14]$. L'existence dans cet oncogène de domaines de type HLH (helix-loophelix), leucine zipper et de séquences rappelant des motifs de localisation nucléaire a fait supposer un couplage direct avec des facteurs transcriptionnels. En fait, la délétion du domaine HLH, bien loin d'inactiver p95 vav, lui confère un plein pouvoir oncogénique [13], si bien que le rôle réel de ces éléments et la réalité du couplage à des facteurs de transcription restent incertains.

\section{- Ras, un relais universel des tyrosine kinases}

L'intervention de la protéine Ras dans des voies de signalisation dépendant de récepteurs tyrosine kinases a été étendue à d'autres systèmes que ceux détaillés ci-dessus : chez la drosophile, le gène torso, qui code lui aussi pour un récepteur tyrosine kinase, est couplé, en aval, au produit des gènes Sos et au gène Ras avec intervention d'une protéine de type Raf-1 [15]. L'insuline, activant l'autophosphorylation sur des tyrosines du récepteur auquel elle est liée, semble nécessiter, pour agir, la présence d'une protéine Ras fonctionnelle [16]. La différenciation de fibroblastes 3T3-L1 en adipocytes peut être provoquée par traitement à l'insuline ou par transfection à l'aide d'un vecteur commandant la synthèse d'une protéine Ras oncogénique [17].

Les cytokines hématopoïétiques de la famille des interleukines (IL2, IL3, IL5) et du GM-CSF (granulocytemacrophage colony-stimulating factor) se fixent sur des récepteurs à un seul passage transmembranaire dénués, par eux-mêmes, d'activité tyrosine kinase [18]. La réponse à ces cytokines dépend, elle aussi, des proto-oncogènes Ras, via l'activation très probable de tyrosine kinases intracellulaires [19] $\mathrm{m} / \mathrm{s} n^{\circ} 5$, wol. 8, mai 92

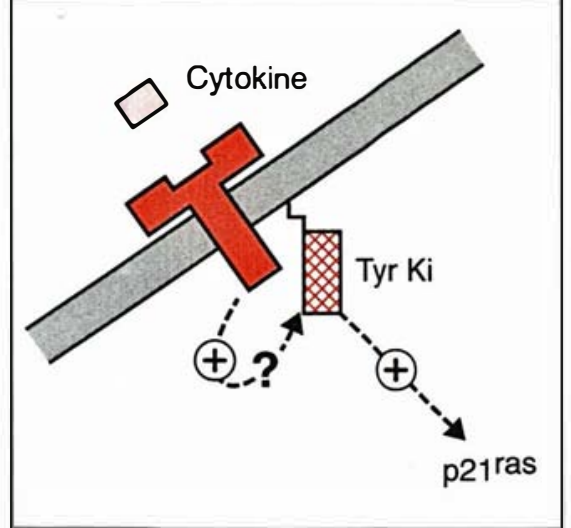

Figure 2. Mécanisme du probable couplage entre les récepteurs des cytokines hématopoïétiques et p21ras. Les récepteurs des facteurs de croissance hématopoiétiques n'ont pas d'activité tyrosine kinase propre. IIs pourraient être couplés, par un mécanisme encore inconnu, avec des tyrosines kinases intracellulaires dont l'activation passerait, comme cela est présenté dans la figure 1, par l'intermédiaire du système p21ras. Ce couplage pourrait être direct, ou se faire par l'intermédiaire de protéines de type Sem 5 ou p95vav.

(figure 2). En d'autres termes, le couple formé par un récepteur de cytokine hématopoḯtique et une tyrosine kinase intracellulaire de type $\mathrm{p} 60^{\text {src }}$ ou $\mathrm{p} 56^{\text {lck }}$ serait l'équivalent fonctionnel des récepteurs de facteurs de croissance dotés, par eux-mêmes, d'une activité de tyrosine kinase.

Le rôle de relais joué par p $21^{\text {ras }}$ a été également démontré dans l'activation des lymphocytes $\mathrm{T}$, secondaire à la reconnaissance du récepteur de ces cellules par l'antigène spécifique [20]. Dans ce cas, on ne sait pas si l'activation de tyrosine kinases de type p56 $6^{\text {Ick }}$ ou celle de la protéine kinase $\mathrm{C}$ sont des intermédiaires entre l'activation du récepteur et la mise en jeu de Ras.

GAP, effecteur et (ou) régulateur négatif de Ras

L'ensemble des résultats que nous venons de commenter est parfaitement démonstratif quant aux relations entre les voies des récepteurs de facteurs de croissance et de cytokines et les protéines p21 ras. En revanche, persistent une incertitude et des inconnues. L'incertitude concerne le rôle réel des protéines GAP. Les travaux du groupe de McCormick montrant que la protéine p120-GAP était nécessaire au couplage entre $\mathrm{p} 21^{\text {ras }}$ et les canaux potassiques de l'oreillette cardiaque $(\mathrm{m} / \mathrm{s}$ $n^{\circ} 4$, vol. 8, p. 388) tendent à faire de GAP, non seulement un régulateur négatif, mais aussi une protéine Ras cible du complexe actif Ras-GTP. En revanche, les travaux génétiques portant sur la voie de la différenciation des photorécepteurs R7 chez la drosophile identifient la protéine Gap-1 comme, uniquement, un régulateur négatif de la transduction du signal par Ras. Très récemment, F. Schweighoffer et al., de l'équipe de B. Tocqué (Rhône Poulenc Rorer, Vitry-surSeine, France) ont obtenu des résultats confortant la vision selon laquelle p120-GAP est nécessaire à la transmission du signal passant par Ras [21]. Ces auteurs ont choisi comme modèle expérimental la transactivation d'un gène test dont le promoteur contient quatre séquences PEA1 dérivées du enhancer du virus du polyome. Le motif PEA1 fixe un complexe AP1, c'est-àdire un hétérodimère Jun/Fos. Par un mécanisme encore inconnu, l'activation de Ras aboutit à la stimulation de la transcription de cette construction génique. L'équipe des laboratoires RhônePoulenc Rorer a tout d'abord montré que la transactivation pouvait être produite par l'introduction dans des cellules, en même temps que le gène test, de constructions codant pour l'oncogène viral $v$-Src, l'oncogène moyen $T$ du virus polyome et la protéine SDC 25 équivalent à un facteur d'échange de levure. v-sarc est une tyrosine kinase, moyen $\mathrm{T}$ agit en activant la tyrosine kinase endogène p60 sic et SDC 25 provoque l'échange du GDP par du GTP, c'est-à-dire l'activation de Ras. Schweighoffer et al. ont ensuite montré que l'introduction supplémentaire d'un plasmide codant pour la région carboxyterminale de GAP, dépourvue des domaines $\mathrm{SH} 2$ et $\mathrm{SH} 3$, bloquait la transactivation du gène test provoqué par tous les moyens que nous venons d'énumérer, mais non pas celle entraînée par l'introduction de l'oncogène $v$-Mos. L'oncogène Mos code pour une sérine thréonine kinase dont la voie de signalisation ne passe, par conséquent, pas par les protéines p21 ras. Le fragment carboxyterminal 
de p120-GAP correspond à la région d'interaction avec le complexe RasGTP et au domaine catalytique responsable de l'activation de l'activité GTPasique. Cependant, l'effet de ce fragment de GAP n'est pas expliqué uniquement par la désactivation de Ras-GTP en Ras-GDP puisque elle s'exerce également sur un Ras oncogénique qui a perdu son activité GTPasique intrinsèque (figure 3 ). Bien plus probablement, il y a compétition pour la fixation à Ras-GTP entre p120-GAP endogène et le fragment carboxyterminal de GAP, ce dernier étant incapable de transmettre le signal plus en aval. En accord avec cette hypothèse, l'inhibition due au fragment GAP carboxyterminal peut être levée en provoquant la synthèse dans la cellule d'une grande quantité de protéine GAP normale. Ces résultats indiquant que p120-GAP est bien située en aval de Ras-GTP dans la transmission du signal n'expliquent en rien les résultats obtenus chez la drosophile chez laquelle l'absence de Gap-1 aboutit à une transmission constitutive du signal et non pas à son arrêt. En réalité, plusicurs types de protéines douées d'activité GTPase-activating protein pourraient exister, les unes et les autres étant des régulateurs négatifs, certaines seulement étant, de plus, des cibles intermédiaires de Ras-GTP. Chez les mammifères, on connaît plusieurs protéines ayant des activités GAP : outre p120-GAP, la protéine NF1, produit du gène de susceptibilité à la neurofibromatose de type 1, a une activité GAP extrêmement puissante pour les protéines p21 ras [22-24]. p120-GAP et NF1 ont en commun le domaine catalytique mais NF1 ne possède pas les domaines $\mathrm{SH} 2$ et $\mathrm{SH} 3$, dont les travaux du groupe de McCormick et ceux de Schweighoffer et al. démontrent qu'ils sont indispensables à la transmission du signal. Peut-être, de ce fait, NF1 est-elle une protéine GAP du même type que Gap-1 de drosophile, c'est-à-dire dont l'action est de limiter la transduction du signal engendré par l'activation de Ras. En faveur de cette hypothèse, on peut relever que NF1 est capable d'inhiber l'action oncogénique de Ras activé, comme le fait le fragment carboxyterminal de p120-GAP doté de son pouvoir d'acti-

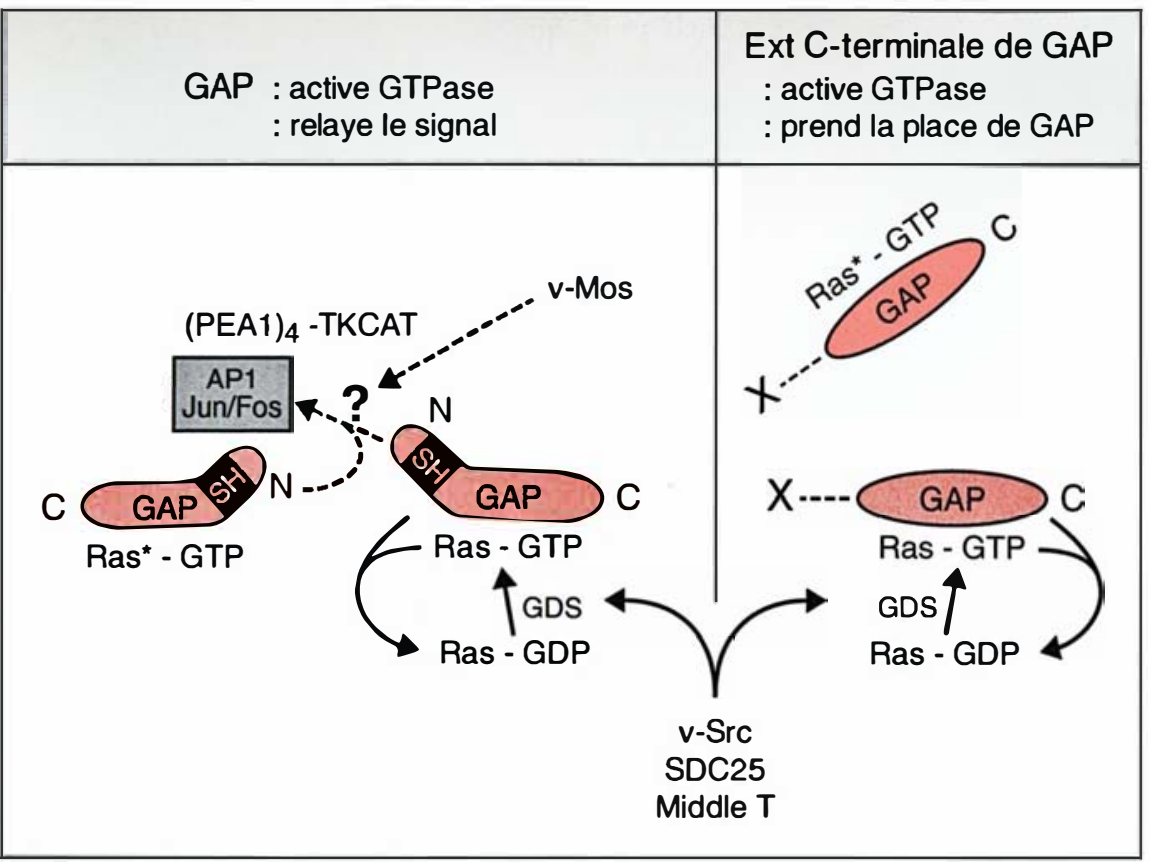

Figure 3. Inhibition de la transactivation empruntant la voie de p21 ras par un fragment carboxyterminal de GAP. Un gène test est constitué de la séquence codant pour la chloramphénicol-acétyl-transférase bactérienne, contrôlée par un promoteur du gène de la thymidine kinase du virus Herpes simplex stimulé par quatre répétitions de l'élément PEA 1. Ce dernier, tiré du enhancer du virus polyome, fixe un complexe AP1, c'est-à-dire un hétérodimère Jun/Fos. En présence de protéine GAP normale, Ras-GTP, normal ou oncogénique, transactive le gène test. Les oncogènes $v$-Src, moyen $T$ de polyome et le facteur d'échange SDC 25 aboutissent également à la transactivation du gène test. Cette transactivation est bloquée par la synthèse dans la cellule de grande quantité d'un fragment carboxyterminal de p120-GAP. Ce dernier agit très probablement par l'intermédiaire d'une compétition avec la protéine GAP entière dont l'extrémité $\mathrm{N}$-terminale serait indispensable à la transmission du signal aboutissant à la transactivation de la construction (PEA 1) \& TK CAT. En revanche, l'oncogène v-Mos, une sérine-thréonine-kinase, continue d'être transactivateur en présence de l'extrémité carboxyterminale de GAP. (D'après les travaux de Schweighoffer et al. [21]).

de son domaine aminoterminal effecteur [24]. Un tel rôle de NF1 s'intégrerait bien dans sa fonction antioncogénique puisque son inactivation entraîne le développement de neurofibromes*.

Reste maintenant à répondre aux questions non résolues par tous ces travaux : quelles sont les étapes de transfert du signal situées en aval de p120-GAP, la couplant aux cibles ulti-

- Trois articles réconls, doux publiés dans le numcio du 17 avril de Cell et le troisème dars celui du 23 avril de Nature établissent une claire conelation entre ure très faible activité de GAP-NF1 [28, 29], due à l'assaciation d'ure oumalie constitusinorde et de mutations acquises [30], et la croissance des cellules de Schwanomes malins $[28,29]$ dars lesquelles, malgré la présence normale de p120-GAP, la proportion de Ras-GTP est élevée. De plus, des mutations ponctuelles du gìne NFI sont égalament observées dans des tumours non relices à la neurofibromalose [30]. mes des signaux engendrés par des facteurs de croissance ou des cytokines? Comment des voies de signalisation aussi différentes et aux effets aussi divers que celles stimulées par l'EGF, le NGF [25], la thrombine [26] ou le TGF $\beta$ [27] peuvent-elles passer par un relais commun? En d'autres termes, quels sont, en aval de l'activation de Ras, les mécanismes de la diversification et de la spécialisation des signaux ? Voilà bien des réponses en attente qui seront probablement apportées par cette combinaison magique de travaux convergents de biochimie chez les mammiferes et de génétique sur des modèles aussi éloignés des précédents que ceux des insectes et des nématodes. Cette possibilité de reconstruire le puzzle à partir de données aussi disparates témoigne de l'unicité des móca- 
nismes en cause, de leur extraordinaire conservation au cours de l'évolution : c'est que nous sommes là probablement à un centre névralgique de la signalisation des cellules vivantes, au moins chez les eucaryotes, là où convergent, sont traités et acheminés de multiples signaux informant la cellule sur son environnement

Axel Kahn

\section{RÉFÉRENCES}

1. De Gunzburg J. Les petites protéines G. médecine/sciences $1992: 8: 322-3$.

2. Goud B. Le transport vésiculaire des cellules eucaryotes est contrôlé par des GTPase. médecine/sciences $1992 ; 8: 324-34$.

3. Bonfini L, Karlovitch CA, Dasgusta C, Banerjee $\mathrm{V}$. The son of sevenless gene product: a putative activator of Ras. Science 1992 ; 255 : 6036.

4. Fortini ME, Simon MA, Rubin GM. Signalling by the sevenless protein tyrosine kinase is mimicked by Ras 1 activation. Nature 1992 ; 355 : 559-61.

5. Gaul U, Mardon G, Rubin GM. A putative Ras GTPase activating protein acts as a negative regulatory of signalling by the Sevenless receptor tyrosine kinase. Cell $1992 ; 68$ : 1007-19.

6. Carthew RW, Rubin GM. Seven in absen$t i a$, a gene required for specification of R7 cell fate in the drosophila eye. Cell 1990 ; 63 : 561-77.

7. Hariharen IK, Carthew RW, Rubin GM.

The Drosophila roughened mutation : activation of a Rap Homolog disrupts eye development and interferes with cell determination. Cell $1991 ; 67: 717-22$

8. Aroian R V, Koga M, Mendel JE, Oshima Y, Steinberg PW. The let-23 gene necesary for Caenorhabitis elegars vulval induction encodes a tyrosine kinase of the EGF receptor subfamily. Nature 1990 ; 348 : 693-9.

9. Beitel CJ, Clark SG, Horvitz RH. Caenorhabitis elegans ras gene let-60 acts as a switch in the pathway of vulval induction. Nature 1990 ; 348 : 503-9.

10. Clark SG, Stern MJ, Horvitz RH. Caenorhabitis elegans cell-signalling gene sem-5 encodes a protein with $\mathrm{SH} 2$ and $\mathrm{SH} 3$ domains. Nature $199 ; 356: 340-4$.

11. Filhol $\mathrm{O}$, Cochet $\mathrm{C}$. Le transfert des signaux mitogéniques : une affaire de particules. médecine/sciences $1990 ; 6: 980-4$.

12. Wood KW, Sarnecki C, Roberts TM, Blenis J. ras mediates nerve growth factor receptor modulation of three signal-transducing protein kinases: MAP kinase, Raf 1 and RSK. Cell 1992; 68 : 1041-50.

13. Bustelo XR, Ledbetter JA, Barbacid M. Product of vav proto-oncogene defines a new class of tyrosine protein kinase substrates. Nature 1992 ; 356 : 68-71.

14. Margolis B, Hu P, Katzav S, et al. Tyrosine phosphorylation of vav protooncogene product containing SH2 domain and transcription factor motifs. Nature $1992 ; 358$ : 71-4.
15. Barinaga. Signals into unknown territory. Science 1992 ; 255 : 1640-1.

16. Burgering BMTh, Medema R, Maassen $\mathrm{JA}$, et al. Insulin stimulation of gene expression mediated by 221 ras activation. EMBO J $1991 ; 10: 1103-9$.

17. Benito M, Porras A, Nebreda AR, Sanlos E. Differentiation of 3T3-L1 fibroblasts to adipocytes induced by transfection of ras oncogenes. Science $1991 ; 253$ : 565-8.

18. Wendling F, Tambourin P. La superfamille des récepteurs de cytokines et l'oncogène $v$-mpl. médecine/sciences $1991 ; 7$ : 569-77.

19. Duronio V, Welham MJ, Abraham S, Dryden P, Schrader JW. p21 ras activation via hemopoietin receptors and c-kit requires tyrosine kinase activity but not tyrosine phosphorylation of $\mathrm{p} 21^{\text {ras }}$ GTPase activating protein. Proc Natl Acad Sci USA $1992 ; 89: 1587$.

20. Downward J, Graves JD, Warne PH, Rayter S, Cantrell DA. Stimulation of p21 ras upon T-cell activation. Nature $1990 ; 346$ : 719-23.

21. Schweighoffer F, Barlat I, ChevallierMulton Mc, Tocqué B. Implication of Gap in Ras-dependent transactivation of a polyoma enhancer sequence. Science $1992 ; 256$ : 825-7.

22. Xu G, Lin B, Tanaka K, et al. The catalytic domain of the neurofibromatosis type 1 gene product stimulates Ras GTPase and complements ira mutants of $S$. cerevisiae. Cell $1990 ; 63$ : 835-41.

23. Martin GA, Viskochil D, Bollag G, et al. The GAP-related domain of the neurofibromatosis type 1 gene product interacts with Ras p21. Cell $1990 ; 63$ : 843-9.

24. Ballester R, Marchuk D, Boguski M, et al. The NF1 locus encodes a protein functionnaly related to mammalian GAP and yeast IRA proteins. Cell $1990 ; 63: 851-9$.

25. Chao MV. Growth factor signaling: where is the specificity? Cell 1992; 68: 995-7.

26. Cichowski K, McCormick F, Brugge JS. p21 ras GAP association with Fyn, Lyn and Yes in thrombin-activated platelets. J Biol Chem $1992 ; 267$ : 5025-8.

27. Mulder KM, Morris SL. Activation of p21 ras by transforming growth factor $\beta$ in epithelial cells. J Biol Chem 1992; 267 : 5029-31.

28. De Clue JE, Papageorge AG, Fletcher $\mathrm{JA}$, et al. Abnormal regulation of marnmalian p21 ras contributes to malignant tumor growth in von Recklinghausen (type 1) neurofibromatosis. Cell $1992 ; 69$ : 265-73.

29. Basu TN, Gutmann DH, Fletcher JA, et al. Aberrant regulation of ras proteins in malignant tumour cells from type 1 neurofibromatosis patients. Nature $1992 ; 356$ : 713-5.

30. Ly Y, Bollag G, Clark R, et al. Somatic mutations in the neurofibromatosis 1 gene in human tumors. Cell $1992 ; 69: 275-81$. 